\title{
Preface
}

\section{Enhanced Recovery and the \\ Changing Landscape of Major Abdominal Surgery}

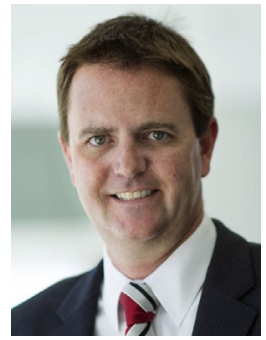

Timothy E. Miller, MB ChB, FRCA

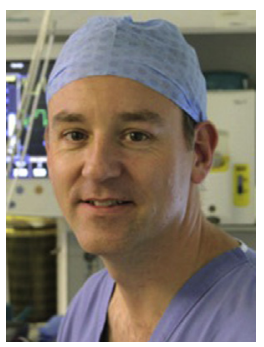

Michael J. Scott, MB ChB, MRCP, FRCA, FFICM

Editors

This issue of Anesthesiology Clinics focuses on the anesthetic management and perioperative care pathway for patients undergoing major abdominal surgery. The last decade has seen increasing recognition that a properly planned and managed perioperative care pathway can improve outcomes after major surgery. There has also been widespread adoption of minimally invasive surgical techniques (both laparoscopic and robotic) that when effectively utilized have contributed to accelerated recovery by minimizing surgical injury. When both are done well, it leads to a very short length of stay for surgical procedures that were once performed using open surgical techniques with high morbidity and mortality.

The development of Enhanced Recovery Pathways (ERPs), first in colorectal surgery and now increasingly across all elective surgical specialties, has lead to the development of patient-focused multidisciplinary teams consisting of anesthesiologists, surgeons, physicians, nurses, physiotherapists, and dieticians, all of whom contribute to improving the outcome for patients. ERPs aim to deliver evidence-based practice (and practice-based evidence) across a multitude of treatment elements to reduce the stress response and improve the metabolic response to major surgery. There is increasing evidence this in turn shortens length of stay, reduces complications and costs, and improves long-term outcomes. The ERAS Society has been the first to publish guidelines in a variety of specialties with the aim of reviewing and updating the evidence base in three yearly cycles. Already we have seen significant shifts in evidence base, such as the move away of using epidural anesthesia in laparoscopic colorectal surgery. Medicine moves fast and we must all move with it!

In this issue, we have started with two articles focusing on prehabilitation and assessment for major surgery using cardiopulmonary exercise testing. We then have 
an article that outlines the effects an ERP has on the pathophysiology during major surgery, before moving on to articles on abdominal surgical specialties, with the emphasis on surgery within modern ERAS protocols. There are specific articles on fluids and analgesia because they are the two key elements that are delivered by anesthesiologists that, combined with minimally invasive surgery, have a major effect on producing optimal outcomes. We end with an article on Emergency Surgery, which is an area that until recently patients have been very poorly served by, but there are exciting developments with pathways improving care-combining Enhanced Recovery and Surviving Sepsis principles.

All articles in this issue have contributions by experts in their field and eminent anesthesiologists with experience and who have driven changes in the perioperative care pathway. It is now time for anesthesiologists to recognize that they don't just have the best understanding of the perioperative care pathway but are in the perfect position to deliver most of the key factors essential for optimal surgical outcomes. They should embrace their new role as Anesthesiologist and Perioperative Care Physician.

\section{Timothy E. Miller, MB ChB, FRCA Associate Professor} Department of Anesthesiology Duke University Medical Center Durham, NC 27710, USA

Michael J. Scott, MB ChB, MRCP, FRCA, FFICM Department of Anesthesia and Perioperative Medicine Royal Surrey County Hospital NHS Foundation Trust Guildford, Surrey GU1 7XX, UK

Surrey Peri-operative Anesthesia Critical care Research Group (SPACeR) Faculty of Health and Medical Sciences

University of Surrey Guildford, Surrey GU2 7XH, UK

E-mail addresses: timothy.miller2@duke.edu (T.E. Miller) mjpscott@btinternet.com (M.J. Scott) 